\title{
El papel de las TIC's en el desarrollo local. Caso: Microempresas en proyectos de extensión. Sede Regional Brunca
}

\author{
The Role of ICTs in Local Development. Case: \\ Microenterprises in Extension Projects. Brunca \\ Regional Campus
}

\author{
Melvin Bermúdez Elizondo \\ Universidad Nacional \\ Sede Regional Brunca, \\ Heredia, Costa Rica \\ melvin.bermudez.elizondo@una.cr \\ Hairol Romero Sandi \\ Universidad Nacional \\ Sede Regional Brunca \\ Heredia, Costa Rica \\ hairol.romero.sandi@una.cr
}

Recibido: 16/02/2018 Aceptado: 18/05/2018

Resumen. Desde los años 80's algunos autores como Michael Porter, visualizaban el papel preponderante que tendrían las Tecnologías de Información y Comunicación (TIC's) y los procesos de innovación en el desarrollo de la competitividad empresarial. El presente trabajo abarca el rol que juegan las TIC's en el desarrollo local, sobre la base que las empresas que se encuentran en las regiones han de tener sistemas de información que les contribuya en un proceso racional de toma de decisiones, dado que sin información certera y oportuna las empresas locales no podrán ser competitivas con relación a las empresas transnacionales y multinacionales. Se exponen los resultados de un caso de estudio con relación a las empresas que han participado en el proyecto de extensión de la Sede Regional 
Revista Universidad en Diálogo • Vol. 8, N. 2, Julio-Diciembre, 2018, pp. 25-37

ISSN 2215-2849 • EISSN: 2215-4752

DOI: http://dx.doi.org/10.15359/udre.8-2.2

Brunca de la Universidad Nacional de Costa Rica, denominado "Fortalecimiento de la Competitividad de diez microempresas del cantón de Pérez Zeledón, Región Brunca". Se concluye que las TIC's juegan un papel preponderante en el desarrollo del parque empresarial de la Región Brunca y por lo tanto son pilar fundamental en el desarrollo local y regional.

Palabras claves: desarrollo, competitividad, microempresas, proyectos, sistemas de información, TIC's.

Abstract. Since the 1980s some authors like Michael Porter visualized the preponderant role that Information and Communication Technologies (ICTs) and innovation processes would have in the development of business competitiveness. This paper covers the role that ICTs play in local development, based on the fact that companies located in the regions must have information systems that contribute to a rational decision-making process, given that without accurate and timely information local companies cannot be competitive in relation to transnational and multinational companies. Here are presented results from a case study about companies that have participated in the extension project of the Sede Regional Brunca of the Universidad Nacional (Brunca Regional Campus of the National University), called "Strengthening the Competitiveness of ten microenterprises in the Pérez Zeledón County, Brunca Region." It is concluded that ICTs play a preponderant role in the development of the business park of the Brunca Region and, therefore, are a fundamental pillar in local and regional development.

Keywords: development, competitiveness, microenterprises, projects, information systems, ICTs.

\section{Introducción}

En los últimos años la sociedad civil ha estado demandando una mayor presencia proactiva de las universidades públicas dentro del ámbito productivo y social. Y si se hace un contraste con la realidad vigente en la Región Brunca, donde diversos diagnósticos regionales han demostrado la necesidad de que las organizaciones productivas aumenten, se requiere potenciar la competitividad por medio de un fortalecimiento de la gestión técnico-administrativa que realizan, ya que se han detectado debilidades en esta gestión que limitan su crecimiento empresarial. Esta iniciativa nació de dos académicos de la Universidad Nacional (UNA), Sede Regional Brunca, Campus Pérez Zeledón en Costa Rica. 
Abordar ambas áreas, las TIC y la administración, hizo de este proyecto un baluarte en la pertinencia de su aplicación en diez microempresas de la Región Brunca, y con ello se promovió una de las áreas que tanto critican de las universidades públicas, la extensión. Y es precisamente la extensión el punto de partida de este proyecto innovador en Costa Rica: colaborar con estas microempresas para que puedan mejorar la gestión gerencial, administrativa y técnica abarcando dos áreas de vital importancia en estos tiempos cada vez más cambiantes y competitivos.

Las diez microempresas fueron debidamente seleccionadas por presentar problemas técnico-administrativos importantes, se les realizó un estudio minucioso para conocer la realidad en que se encontraban y poder así ser parte del proyecto. Este estudio tuvo como punto inicial conocer todo lo concerniente a cada microempresa y así poder potenciar un aprovechamiento de la capacidad instalada a nivel de infraestructura, un uso eficiente de las tecnologías de información y comunicación, una mayor cantidad de encadenamientos productivos, un alto estándar de calidad, una mayor diversificación y un valor agregado de los productos, con una mayor capacidad para la presentación de los proyectos, todo ello para que repercuta en menores costos de producción, mayores ventajas competitivas y por ende en un crecimiento de la competitividad.

\section{Objetivos}

1. Implementar sistemas de gestión y control administrativo en el manejo gerencial de las microempresas, sean estos de tipo manual o automatizado.

2. Desarrollar e implementar paquetes informáticos y manuales de software específico para las microempresas, además de disponer de los manuales prácticos de los paquetes y del software

\section{Marco conceptual}

\section{El papel de las TIC en las organizaciones}

Desde los años ochenta algunos autores como Michael Porter visualizaban el papel preponderante que tendrían las tecnologías de información y comunicación (TIC) y los procesos de innovación en el desarrollo de la competitividad empresarial (Porter, 1996). 


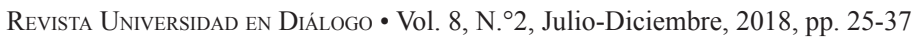

ISSN 2215-2849 • EISSN: 2215-4752

DOI: http://dx.doi.org/10.15359/udre.8-2.2

Antes de iniciar con la explicación del papel que desempeñan las TIC en las organizaciones, es necesario definir algunos conceptos relevantes:

\section{Mipymes}

El término mipymes hace referencia a las empresas consideradas micro, pequeñas y medianas, tomando en cuenta algunas particularidades específicas que caracterizan la operación de estos negocios. Múltiples autores y autoras han expresado definiciones para la empresa, teniendo en consideración distintos elementos que se consideran relevantes para describir a esa unidad productiva generadora de un servicio o producto con la perspectiva de satisfacer la necesidad existente de una tercera persona y de esta manera obtener a cambio un beneficio monetario en la mayoría de las ocasiones.

En este trabajo se utilizará la siguiente definición, contenida en el Reglamento general de la Ley $\mathrm{N}^{\circ} 8262$ de Fortalecimiento de las Pequeñas y Medianas Empresas (Asamblea Legislativa, 2002) que en su artículo 3 inciso g establece: "PYME: Unidad productiva de carácter permanente que dispone de recursos físicos estables y de recursos humanos; los maneja y opera, bajo la figura de persona física o persona jurídica, en actividades industriales, comerciales o de servicios.

TIC

TIC es la sigla del término tecnologías de la información y la comunicación. Existen muchas definiciones de este término tan actual, entre las que se puede mencionar la siguiente:

TIC: En líneas generales podríamos decir que las nuevas tecnologías de la información y comunicación son las que giran en torno a tres medios básicos: la informática, la microelectrónica y las telecomunicaciones; pero giran, no sólo de forma aislada, sino lo que es más significativo de manera interactiva e interconexionadas, lo que permite conseguir nuevas realidades comunicativas (Cabero, 1998).

Lo que da a entender este término es el impacto que conlleva contar con las TIC en las organizaciones, ya que promueven nuevas formas o realidades comunicativas, sin dejar de lado el gran beneficio que conllevan, que es el mejoramiento de los procesos administrativos en las mismas organizaciones al contar con toda una infraestructura tecnológica apropiada. 
Teniendo claro ambos conceptos relevantes para este proyecto, es necesario explicar el papel preponderante de las TIC en el desarrollo local de las mipymes y en especial el impacto que tienen al promover y aumentar la competitividad en el mercado local, regional, nacional e internacional.

\section{Desarrollo local}

En los últimos años en Costa Rica han surgido gran cantidad de microempresas, las cuales constituyen una fuente fundamental para el desarrollo socioeconómico. Y si se gestionan por medio de las TIC, estas organizaciones podrán contar con mayores oportunidades de ser competitivas.

Es por ello que es importante promover el surgimiento y aumento de la competitividad de estas empresas, ya que son un medio para aumentar la fuente de empleo, principalmente en las zonas rurales, que es donde se perciben mayores necesidades económicas en las familias, debido a las pocas oportunidades de estudio y preparación que presentan y sobre todo por los limitados recursos que tienen.

En términos locales, específicamente en el cantón de Pérez Zeledón, la importante participación de las mipymes en la economía no difiere de la realidad nacional costarricense. Incluso utilizando la estadística de la CCSS, que determina la cantidad de empleos generados como la base para establecer si una empresa se clasifica como micro, pequeña o mediana, el 99\% de los/ as patronos/as inscritos/as ante la CCSS administran una mipyme, generando empleo formal a un total de 7.402 habitantes de Pérez Zeledón (Segura, 2012).

Las diez mipymes involucradas en la realización del proyecto pasaron por una serie de etapas de selección y priorización que no serán abarcadas en este trabajo. En cambio, sí se abarcará el marco de la extensión universitaria por parte de las personas participantes, de la comunidad académica y estudiantil de las dos áreas involucradas, ingeniería en sistemas y administración.

\section{Marco metodológico}

Se debe indicar que el principio del proyecto tuvo un alto grado de investigación cualitativa, complementado con varios diagnósticos regionales realizados por la Oficina Regional del Ministerio de Economía, Industria y Comercio (MEIC, 2012), la Oficina Regional de Procomer y de las Oficinas Regionales del MAG (que constituyen la Red de Apoyo Regional a las Pymes), donde se 
ReVista Universidad en Diálogo • Vol. 8, N. ${ }^{\circ}$ 2, Julio-Diciembre, 2018, pp. 25-37

ISSN 2215-2849 • EISSN: 2215-4752

DOI: http://dx.doi.org/10.15359/udre.8-2.2

determinó que las organizaciones productivas de la Región Brunca requieren aumentar la competitividad por medio del fortalecimiento de la gestión técnico-administrativa que realizan, ya que se han detectado debilidades en esta gestión que limitan su crecimiento empresarial. De ahí se priorizaron diez microempresas de la Región Brunca para conformar este proyecto.

En cuanto a la investigación cualitativa, se debe mencionar a Hernández, Fernández y Baptista (2010), los cuales señalan que “...la investigación es un conjunto de procesos sistemáticos, críticos y empíricos que se aplican al estudio de un fenómeno". Los mismos autores señalan las tres grandes clasificaciones de la investigación: cuantitativa, cualitativa y la mixta. El enfoque cuantitativo es secuencial y probatorio, representa un conjunto de procesos. El orden de los procesos es riguroso, donde sobresalen los siguientes: la idea, el planteamiento del problema, la revisión de textos, el alcance del estudio, la elaboración de las hipótesis y definición de las variables, el desarrollo del diseño de la investigación, la definición y selección de la muestra, la recolección de datos y su posterior análisis, el análisis de los datos y por último la elaboración de resultados (Hernández et al., 2010).

El tercer enfoque, el mixto, representa un conjunto de procesos sistemáticos, empíricos y críticos de investigación e implican la recolección y el análisis de datos cuantitativos y cualitativos (Hernández et al., 2010). Queda claro que en los métodos mixtos se combinan al menos un componente cuantitativo y uno cualitativo en un mismo estudio o proyecto de investigación.

El tipo de diseño que más se adaptó a este proyecto fue el de investigaciónacción, debido a que es una forma de estudiar y explorar una situación social, con la finalidad de mejorarla, en la que se desenvuelven como indagadoras las personas implicadas en la realidad investigada (Suárez, 2002). Asimismo, John Elliot (2006), uno de los autores más reconocidos en cuanto a este diseño de investigación, señala que la investigación-acción es el "estudio de una situación social con el fin de mejorar la calidad de la acción dentro de la misma".

En este tipo de diseño de investigación es necesario un alto compromiso de las personas participantes o sujetos de información para el proyecto, así como una gran motivación por obtener el resultado esperado, que en este caso fue la implementación de siete soluciones tecnológicas en las diez microempresas seleccionadas para el proyecto de extensión en el Campus de Pérez Zeledón de la Universidad Nacional. 
Con respecto a los sujetos de información o "indagadores", según John Elliot (2006), se enfocarán en dos ambientes, el interno y el externo. El nivel externo estuvo compuesto por las diez organizaciones, sus puestos de alto mando (administrativos) y los/as colaboradores/as (puestos operativos). El interno estuvo conformado por la comunidad universitaria del Campus de Pérez Zeledón, específicamente estudiantes de Administración e Ingeniería en Sistemas, además de los dos académicos involucrados en el proyecto.

\section{El proceso de desarrollo de software}

Posteriormente, al contar con los requerimientos informáticos necesarios, cada grupo de estudiantes de la carrera de Ingeniería en Sistemas de Información determinó una serie de fases para desarrollar el software. A continuación, se explica cada una de estas fases según la metodología en cascada, utilizada por la mayoría de los grupos de desarrollo.

El modelo en cascada da las pautas que permiten la organización en el desarrollo del software a través de la implementación de sus fases, esto quiere decir que cuando se estén llevando a cabo todas las tareas pertinentes dentro de esa fase, no se podrá avanzar a la siguiente fase hasta no concluir con todas las tareas (Fox, 1982).

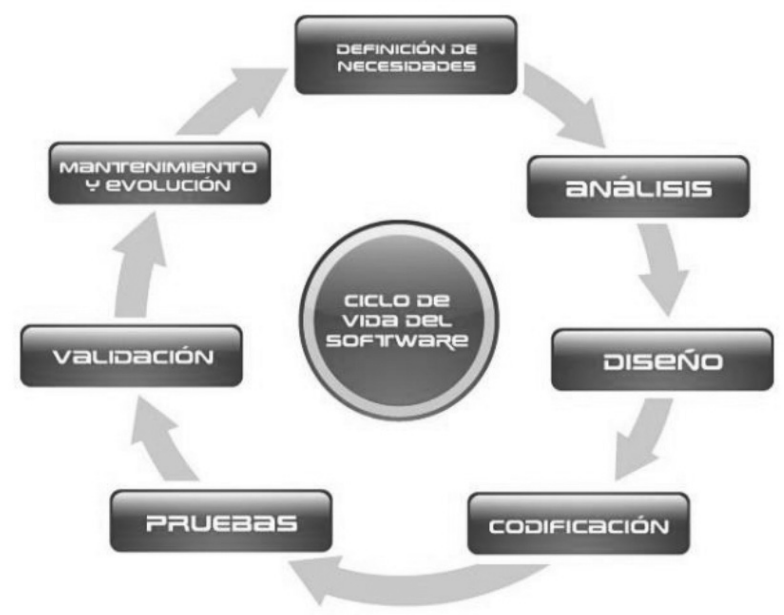

Figura 1. Proceso de Desarrollo de Software.

Fuente: Fox (1982). 
ReVista Universidad en Diálogo • Vol. 8, N. ${ }^{\circ}$ 2, Julio-Diciembre, 2018, pp. 25-37

ISSN 2215-2849 • EISSN: 2215-4752

DOI: http://dx.doi.org/10.15359/udre.8-2.2

Este modelo se compone de distintas fases, a saber: análisis, diseño, programación, pruebas, implantación y capacitación, y finalmente mantenimiento. El análisis a su vez se subdivide en el análisis de requisitos y el análisis del diseño. El diseño del programa se enfoca en determinar el diseño de la solución, para la posterior fase que es la de programación o codificación. Seguida de la fase de pruebas, capacitación e implantación para terminar en la fase final de mantenimiento de la solución informática.

Como bien se indicó previamente, todos los grupos de desarrollo utilizaron esta metodología. Es importante mencionar que la selección de esta metodología se basó en el tipo de proyecto a desarrollar, donde las mismas fases que se describen a continuación son las más apropiadas para los proyectos.

El análisis de requisitos o especificación de características que ha de cumplir el software que se va a desarrollar es la primera fase del modelo en cascada. Y probablemente sea la más importante. Al fin y al cabo, lo que sea o no sea el producto final depende de decisiones tomadas en esta fase. Se trata fundamentalmente de estudiar las necesidades y preferencias de las personas usuarias. Es también muy importante dejar clara constancia de las decisiones tomadas en esta fase, para ser tenidas en cuenta posteriormente (Fox, 1982). Por ello, la documentación producida en esta fase debe ser concreta y estar siempre disponible durante el resto del proceso.

En el diseño se deben analizar las posibles soluciones desde un punto de vista técnico. En el análisis de cada una de las partes nos encontraremos normalmente con que hay varias soluciones posibles y se plantea el diseño del programa a partir de los requisitos. Una de las principales decisiones a tomar en esta fase es la del lenguaje a emplear para desarrollar el proyecto. En el caso específico de las siete soluciones informáticas, se seleccionaron herramientas de software libre que no implican un costo para las organizaciones y tienen fines académicos.

En la codificación se realiza el trabajo de desarrollar o codificar, como bien lo indica el nombre de la fase, el software basado en las fases anteriores y poder así culminar con un producto de software a la medida de cada una de las organizaciones incluidas en este proyecto de extensión.

Como parte de la codificación, y aunque se ve como una fase posterior, están las pruebas. En esta fase hay que comprobar que las especificaciones o requisitos se cumplan perfectamente y en todos los casos (Fox, 1982). Esta fase se realizó en conjunto con las personas que colaboraron en cada una 
de las organizaciones, para poder depurar el software y que este llegue a ser la solución adecuada para colaborar con cada una de las organizaciones.

Una vez realizada la respectiva depuración del software con las pruebas, se continúa con la capacitación respectiva y posterior implementación del producto en las organizaciones. En esta fase es importante separar ambos aspectos. En primera instancia se realizaron capacitaciones in situ, es decir, una vez instalado el software la persona colaboradora que será la usuaria directa en el uso del software realiza una capacitación donde se abarquen y resuelvan dudas que puedan surgir en el proceso de capacitación. Una vez realizadas las respectivas capacitaciones, se llega a la fase de entregar el software listo y con la configuración necesaria para su utilización.

\section{Resultados}

Como bien se ha indicado a lo largo de este trabajo, las TIC colaboran y potencializan el desarrollo local de las organizaciones por medio del desarrollo e implementación de las soluciones informáticas y para las que estuvieron involucradas en este proyecto no fue la excepción.

En este apartado se presenta de forma general cada uno de los software desarrollados para cada una de las organizaciones involucradas en este proyecto de extensión.
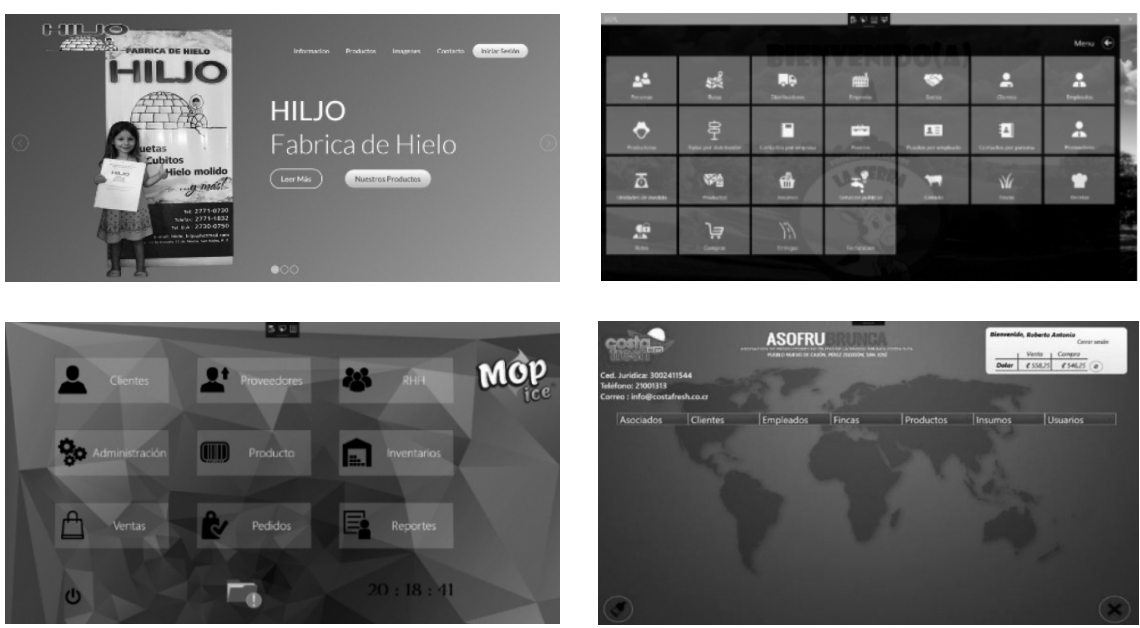

Figura 2. Elaboración de Software para el Proyecto de Extensión 
Todos los sistemas fueron desarrollados con un alto grado de calidad y manejo de errores, donde el colaborador o la colaboradora que utilice el sistema (usuario/a) pueda conocer de primera mano cuáles son los pasos a seguir, esto gracias a una interfaz intuitiva y agradable, de fácil manejo y que responde a las solicitudes de forma eficiente a nivel de tiempos de respuesta, todo bajo el cumplimiento de los requerimientos que las mismas personas usuarias solicitaron al inicio del proyecto.

Los sistemas corresponden a las siguientes organizaciones:

- Hielos HILjO, que es una empresa dedicada a la fabricación de hielo en la Zona Sur del país y con un alto compromiso social. HILJO se rige bajo las normas de higiene y salud, cuenta con la mejor maquinaria especializada para el diseño del producto que ofrece. El hielo que produce es único en la Zona Sur, ya que utilizan un mejor proceso que hace que el hielo sea más puro y de mucha mayor duración. El software desarrollado está enfocado en la gestión financiero-administrativa de dicha organización. Esta solución fue la única que se enfocó en un ambiente web por la alta disponibilidad del equipo tecnológico con el que contaba la empresa. Las demás soluciones por solicitud de las mismas organizaciones fueron para un ambiente de escritorio.

- MOPICE, empresa pionera en la fabricación de refrescos. El producto cuenta con una gran aceptación comercial y su mercado meta es la población más joven en escuelas y colegios del país, además de la colocación del producto en pulperías. El software desarrollado está enfocado en la gestión financieroadministrativa de dicha organización.

- ASOFRUBRUNCA, que es una empresa agroindustrial. Dicha asociación nace por la gran necesidad de apoyo a los productores y las productoras de frutas en la Zona Sur, en especial del distrito de Cajón de Pérez Zeledón, que es el lugar donde está ubicada la organización. La asociación basa su economía en la fruta llamada rambután, conocida en nuestro país como mamón chino. El software desarrollado está enfocado en la gestión financiero-administrativa de dicha asociación. Este software permite el desarrollo del control administrativo de las ventas internacionales que dicha asociación realiza. 
- COOPESIERRA es una pequeñaempresa dedicada alaproducción y comercialización de leche y sus principales derivados, surge con el esfuerzo de la Asociación Agropecuaria La Sierra de Platanares con el objetivo de mejorar la situación económica de las familias de la comunidad. El software desarrollado está enfocado en la gestión financiero-administrativa de dicha asociación, especialmente en el control de fincas, ganado $y$, por supuesto, la comercialización de la leche y sus principales derivados, que realizan las personas asociadas.

- Almácigos del Sur, que es una pequeña empresa enfocada en la hidroponía con el fin de cubrir el consumo familiar. Al crecer la producción con éxito, se decidió ofrecer a la comunidad, que después también se acercaba en busca del producto. Conforme pasaron los años la pequeña empresa creció a nivel de ventas, convirtiéndose en un proveedor para varios comercios regionales. El software desarrollado está enfocado en la gestión financiero-administrativa de dicha empresa familiar.

Todos los software tienen como finalidad resolver la necesidad operante de las organizaciones, todos los software son de carácter financieroadministrativo, productor-financiero y productor-administrativo.

\section{Discusión y conclusiones}

Como parte de las conclusiones más importantes y eventuales discusiones que deja la culminación del proyecto de extensión, se mencionan las siguientes:

- Estudiantes: Un alto enriquecimiento en experiencia y conocimiento al desarrollar una solución informática real para una organización de la zona y con necesidades reales a nivel de tecnologías de información.

- Organizaciones: Lograr la automatización de muchos de los procesos realizados de manera manual y contar con una solución tecnológica que agilice la operación diaria, pero sin dejar de lado el control necesario en la ejecución de los procesos, para lograr un mayor grado de competitividad en el mercado regional, nacional e internacional. 
- Académicos/as: Aportar a las organizaciones de la región una solución técnico-administrativa que venga a potencializar su accionar y la competitividad en un mercado cada vez más cambiante, gracias a la realización de un proyecto de esta magnitud.

- UNA: Realizar extensión a nivel regional en dos áreas importantes en las organizaciones: administración y tecnologías, por medio de un proyecto de extensión que abarcó ambas áreas y desarrolló una solución técnico-administrativa para diez microempresas de la región sur del país, logrando con ello un mejor posicionamiento a nivel de mipymes.

- La importancia de incorporar las TIC en los proyectos de extensión que ejecute la Universidad, puesto que independientemente del área en que se desarrolle el proyecto, las TIC estarán presentes.

A nivel de discusión sobresalen las siguientes consultas que se pueden inferir a partir de este trabajo de extensión:

- ¿De quién es la responsabilidad de incentivar los proyectos de extensión en la UNA? ¿De las autoridades o del personal académico con experiencia en extensión?

- ¿Por qué son pocas las personas participantes en proyectos de extensión? ¿Cuáles son los inconvenientes que se presentan?

- ¿Cuáles son las ventajas de participar en proyectos de extensión para un académico o una académica?

- ¿Cuál creen que es el rol de los y las estudiantes en los proyectos de extensión? ¿Es necesaria su participación?

\section{Referencias}

Asamblea Legislativa. Ley de Fortalecimiento de las Pequeñas y Medianas Empresas (2002). Recuperado de http://www.asamblea.go.cr/ Centro de informacion/Centro Dudas/Lists/Formule\%20su\%20 pregunta/Attachments/677/8262.p.pdf

Cabero, J. (1998). Impacto de las nuevas tecnologías de la información y la comunicación en las organizaciones educativas. En M. Lorenzo 
y otros (coords.), Enfoques en la organización y dirección de instituciones educativas formales y no formales (pp. 197-206). Granada: Grupo Editorial Universitario.

Elliot, J. (2005). La investigación-acción en educación. España: Editorial Morata.

Fox, J. M. (1982). Software and its development. Prentice-Hall. Recuperado de http://dit.upm.es/ fsaez/intl/libro_complejidad/15-el-desarrollodel-software.pdf

Hernández, R., Fernández, C. y Baptista, P. (2010). Metodología de la investigación ( $5^{\mathrm{a}} \mathrm{ed}$.). Editorial McGraw Hill.

Ministerio de Industria y Comercio (MEIC). (2012). Estudio oferta de crédito para la pyme en Costa Rica, 2011. Recuperado de http:// pymes.elfinancierocr.com/sites/default/files/estudio_credito_ pymes.pdf

Política Pública de Fomento a las Pyme y al Emprendedurismo (2010). Recuperado de http://www.pyme.go.cr/media/archivo/normativas/ Politica\%20PubFomentoPYMEEmpre.pdf

Porter, M. (1996). Estrategias competitivas. Técnicas para el análisis de los sectores industriales y de la competitividad. México: Editorial Cecsa.

Porter, M. (2006). Estrategia y ventaja competitiva. Editorial Deusto.

Segura, O. (2012). Realizan encuesta para evaluar desarrollo empresarial de la Región Brunca. Periódico Enlace, edición no 77, p. 17. Recuperado de http://www.gruposiba.com/Enlacecr/enlace\%20 octubre\%202012.pdf

Suárez, M. (2002). Algunas reflexiones sobre la investigación-acción colaboradora en la educación. Revista Electrónica de Enseñanza de las Ciencias, 1(1). 